\title{
Person Death Indicator
}

National Cancer Institute

\section{Source}

National Cancer Institute. Person Death Indicator. NCI Thesaurus. Code $C 93985$.

Specifies whether the person is dead. 